\title{
ETUDE DU COMPORTEMENT ASYMPTOTIQUE DE SOLUTIONS D'ÉQUATIONS DE DIFFUSIONS GÉNÉRALISÉES ET DE PROCESSUS NON GAUSSIENS
}

\author{
SINA AKHBARI, MICHĖLE MASTRANGELO ET BURBUQUE PEPO
}

\begin{abstract}
RÉSUmÉ. Dans cet article, on analyse le comportement asymptotique des solutions des équations de la chaleur d'ordres impairs et rationnels. On utilisera pour cela la méthode dite du "point selle". On étudiera également différents problèmes posés par les "diffusions" non gaussiennes. On s'est inspirés pour cela de problèmes physiques classiques d'hyper ou d'hypo-diffusions.
\end{abstract}

\begin{abstract}
In this article, we analyse the asymptotic behaviour of the fundamental solutions of higher odd or rational order heat-type equations. We will use the "steepest descent " method. We will also study some non gaussian diffusion problems. We will take inspiration from physical classical problems of hyper or hypo-diffusions.
\end{abstract}

\section{IntRoduction}

La motivation principale de ce travail concerne les processus dits "anormaux" car non gaussiens (hypodiffusions ou hyperdiffusions). On est alors amené à des études du type "lois de Lévy", ou les moments d'ordre $\beta \geq 0$ sont de la forme $t^{\frac{\beta}{\alpha}}$ avec $\alpha \neq 2$.

Dans les parties 3 et 4 , nous considérons des équations aux dérivées partielles de la forme:

$$
\frac{\partial u}{\partial t}=c \frac{\partial^{2 n+1} u}{\partial x^{2 n+1}}
$$

avec $n>1, t>0$ et $x$ réel. A cette équation est ajoutée la condition initiale $u(x, 0)=\delta(x)$; la constante $c$ est réelle et $\delta$ dénote la distribution de Dirac. Cette équation est une généralisation de l'équation classique de la chaleur: $\frac{\partial u}{\partial t}=\frac{1}{2} \frac{\partial^{2} u}{\partial x^{2}}$.

L'étude de ce type d'équation a débuté au début des années 1960. On peut citer à ce sujet Krylov (1960), Daletskii $(1962,1969)$ et Ladokhin (1962). L'étude de la première équation a été souvent faite dans la littérature. Les mesures de type Wiener ont été construites au moyen des solutions fondamentales par de nombreux auteurs (Krylov (1960, 1965), Daletskii (1962, 1969), Daletskii et Fomin (1965), Ladokhin $(1962,1965)$, Hochberg (1978), Orsingher (1991), Hochberg et Orsingher (1994))

Une analyse précise du comportement des solutions de $\frac{\partial u}{\partial t}=-\frac{\partial^{4} u}{\partial t^{4}}$ a été faite par Hochberg en 1978 et par Li et Wong en 1993 pour le cas $k$-dimensionel. Plus récemment, on peut citer l'article de Acetta et Orsingher (1997) où est étudiée l'équation $\frac{\partial u}{\partial t}=c \frac{\partial^{2 n} u}{\partial x^{2 n}}$ pour $c=(-1)^{n+1}$. Cet article concerne également l'équation $\frac{\partial u}{\partial t}=(-1) \frac{\partial^{3} u}{\partial x^{3}}$, étudiée par la méthode du chemin de plus grande pente.

(C) Société de Mathématiques Appliquées et Industrielles. Typeset by LATEX.

S. AKHBARI, M. MASTRANGELO, B. PEPO: Université de Paris 6, Mathématiques, 4, Place Jussieu, 75252 Paris cedex 05 (France). 
Il faut pourtant souligner que les intégrales du type:

$$
u(x, t)=\frac{1}{2 \pi} \int \exp \left(i \alpha x+c(i \alpha)^{2 n+1} t\right)
$$

n'ont été étudiées que dans des cas très particuliers avant les années 1960, (Burwell (1923), Bernstein (1919), Lévy (1923), Polya (1923)). Ici, nous étudierons le comportement asymptotique de cette expression pour $n$ entier naturel supérieur ou égal à 2 et $c$ une constante réelle. Il est intéressant de souligner que les résultats ne sont pas symétriques suivant que $x$ tend vers $+\infty$ ou $-\infty$.

Dans la partie 5 nous donnons un aperçu sur l'étude des solutions de l'équation à dérivées fractionnaires, notamment d'ordre $\frac{3}{2}$. Dans les parties 6 et 7 , nous étudions une généralisation de l'intégrale de Wiener, corrélée avec l'équation de diffusion généralisée, et nous regardons les correspondances dans le cas de l'équation de la chaleur.

Enfin, dans la partie 8, nous donnons un aperçu sur l'existence des moments d'ordre non entier.

\section{Notations et définitions générales}

Dans ce travail, nous allons introduire des mesures correspondant à des processus (souvent appelées, abusivement, "diffusions"), non gaussiens; nous nous plaçons pour des raisons de simplification d'écriture, en dimension 1. Nous inspirant des techniques classiques dans le cas brownien, ces densités seront présentées comme des transformées de Fourier d'exponentielles de fonctions de la variable $\lambda^{\alpha} t$, ou plutôt $(i \lambda)^{\alpha} t$, pour $\alpha \in \mathbf{R}_{+}^{*}$

Concernant l'aspect fractionnaire, nous avons adopté un point de vue différent de celui qui est, traditionnellement, proposé pour le mouvement brownien fractionnaire de B. Mandelbröt, présenté dans [9] par M. Giona et H.E. Roman. Dans [9], la dérivée fractionnaire porte sur la variable temporelle:

$$
\frac{\partial^{\mu} u}{\partial t^{\mu}}(x, t)=A \frac{\partial u}{\partial x}(x, t)
$$

alors qu'ici, la dérivée fractionnaire porte sur la variable spatiale:

$$
\frac{\partial u}{\partial t}(x, t)=A \frac{\partial^{\alpha} u}{\partial x^{\alpha}}(x, t) .
$$

Dans le cas de (2.1), la densité de transition est de la forme:

$$
p(x, t)=\frac{K}{t^{1 / \alpha}} \exp \left(-\frac{x^{2}}{k t^{2 / \alpha}}\right),
$$

ce qui conduit à une fonction caractéristique de la forme:

$$
h(t, p)=C \exp \left(-c \lambda^{2} t^{2 / \alpha}\right)
$$

On a alors évidemment, $h(\lambda, t) \cdot h(\lambda, s) \neq h(\lambda, t+s)$, ce qui correspond à

$$
p(., t) * p(., s) \neq p(., t+s) \text {. }
$$

Ce point de vue correspond donc à un abandon de la propriété de semi-groupe de Chapman-Kolmogorov. A contrario, notre version conduit à la conservation naturelle de la propriété de semi-groupe, comme nous le verrons en considérant les fonctions caractéristiques.

Les notions relatives aux transformées de Fourier différant suivant les auteurs, nous choisissons la formulation suivante (en dimension 1):

$$
\mathcal{F} u(\lambda)=(2 \pi)^{-\frac{1}{2}} \int e^{-i \lambda x} u(x) d x
$$




$$
\mathcal{F}^{-1} f(x)=(2 \pi)^{-\frac{1}{2}} \int e^{i \lambda x} f(\lambda) d \lambda .
$$

Nous adoptons en outre la détermination naturelle:

$$
(i \lambda)^{\alpha}=|\lambda|^{\alpha} e^{i \cdot \operatorname{sgn}(\lambda) \cdot \frac{\pi \alpha}{2}},
$$

où $\lambda \in \mathbf{R}$ et où $\operatorname{sgn}(\lambda)=1$ si $\lambda>0$ et $\operatorname{sgn}(\lambda)=-1$ si $\lambda \leq 0$; nous introduisons enfin la notation suivante pour simplifier l'écriture des formules ( $\Re$ désigne la partie réelle d'un complexe):

$$
(i \lambda)^{(\alpha)}=-\operatorname{sgn}\left(\Re\left(i^{\alpha}\right)\right)(i \lambda)^{\alpha}=s_{\alpha}(i \lambda)^{\alpha},
$$

où $s_{\alpha}=\operatorname{sgn}\left(\Re\left(i^{\alpha}\right)\right)$. Cette fonction se prolonge sur $\mathrm{C}$ par: si $\mathrm{z}=\rho e^{i \theta}$ avec $-\pi \leq$ $\theta \leq \pi$, alors $(i z)^{(\alpha)}=-\operatorname{sgn}\left(\Re\left(i^{\alpha}\right)\right) \exp \left(i\left(\frac{\pi}{2}+\theta\right) \alpha\right) \rho^{\alpha}$.

Dans la suite, nous considérons les fonctions suivantes (qui sont, dans certains cas, des fonctions caractéristiques):

$$
h_{\alpha}(\lambda, t)=(2 \pi)^{-\frac{1}{2}} \exp \left[(i \lambda)^{(\alpha)} \frac{t}{\Gamma(\alpha+1)}\right], \alpha>0
$$

où $\Gamma$ est la fonction d'Euler. C'est une fonction caractéristique lorsque $\alpha \leq 2$, d'après un résultat de Levy-Khintchine (voir P. Levy (1954)). On lui associe sa transformée de Fourier réciproque dans l'espace de distributions tempérées:

$$
p_{\alpha}(x, t)=\mathcal{F}^{-1}\left[h_{\alpha}(\bullet, t)\right](x)
$$

Lorsque $\alpha$ est un réel positif non nul qui n'est pas un entier impair, $p_{\alpha}(d x, t)$ est une mesure absolument continue par rapport à la mesure de Lebesgue et nous notons $p_{\alpha}(x, t)$ sa densité; cette fonction est alors de classe $C^{\infty}$ en $(x, t)$ sur $\mathbf{R} \times \mathbf{R}_{+}^{*}$ [23].

Un problème important pour la suite sera l'étude des moments de $p_{\alpha}$ lorsque celle-ci est une mesure.

Nous rappelons quelques propriétés élémentaires de $p_{\alpha}(t)$.

- Pour tout $\alpha \in \mathbf{R}_{+}^{*} \backslash(2 \mathbf{N}+1), \mathcal{F}^{-1}\left(h_{\alpha}(t)\right)$ est une mesure signée réelle de variation totale finie pour $\alpha \geq 1$.

- Elle est absolument continue par rapport à la mesure de Lebesgue pour tout $\alpha \in \mathbf{R}_{+}^{*} \backslash(2 \mathbf{N}+1)$.

- Elle est paire en $x$ si $\alpha \in 2 \mathbf{N}$.

- Pour tout $\alpha \in \mathbf{R}_{+}^{*} \backslash(2 \mathbf{N}+1), p_{\alpha}$ est $C^{\infty}$ sur $\mathbf{R} \times \mathbf{R}_{+}^{*}$;

- Pour tout $\alpha \in \mathbf{R}_{+}^{*} \backslash(2 \mathbf{N}+1), \mathcal{F} p_{\alpha}(x, t)(a)=h(a, t)$.

- Les mesures $p_{\alpha}=p$ vérifient d'autres propriétés de type "gaussien" que nous énonçons pour $\alpha \notin 2 \mathrm{~N}+1$ :

: les mesures $p(x, t) d x$ vérifient la propriété de "scaling":

$$
p\left(\left(\frac{f(t)}{t}\right)^{1 / \alpha} x, f(t)\right)\left(\frac{f(t)}{t}\right)^{1 / \alpha} d x=p(x, t) d x, \text { pour tout } t>0 \text { et } f(t)>0,
$$

ce qui implique, en particulier, $p\left(\left(t^{-2 / \alpha} x, \frac{1}{t}\right) t^{-2 / \alpha}\right) d x=p(x, t) d x$;

: les mesures $p(x, t) d x$ vérifient la propriété de semi-groupe: si $s$ et $t$ sont deux réels strictement positifs, $p(x, t+s)=\int_{y \in \mathbf{R}} p(y, s) p(x-y, t) d y$.

Pour tout réel $s \in \mathbf{R}$, le moment d'ordre $s$ de $p(t)=p_{\alpha}(\bullet, t)$ vérifie:

$$
M_{s}\left(p_{\alpha}\right)=\int|x|^{s} p_{\alpha}(d x, t)=t^{\frac{s}{\alpha}} K_{s}(\alpha),
$$

où $K_{s}(\alpha)$ est une constante (éventuellement infinie) qui ne dépend que de $s$ et de $\alpha$. Utilisant les dérivées non entières, nous allons calculer le générateur infinitésimal du semi-groupe dont le noyau de convolution est $p_{\alpha}(x, t)$. Nous définissons tout d'abord ces notions qui different quelque peu suivant les auteurs. 
Pour tout $\beta \geq 0$, et pour toute fonction $u$ appartenant à $L^{1}(\mathbf{R})$ et telle que $|\lambda|^{\beta} \mathcal{F} u(\lambda)$ appartienne aussi à $L^{1}(\mathbf{R})$, la dérivée partielle $\frac{\partial^{\beta} u}{\partial x^{\beta}}$ est définie par:

$$
\frac{\partial^{\beta} u}{\partial x^{\beta}}(x)=\mathcal{F}^{-1}\left[(i \lambda)^{\beta} \mathcal{F} u(\lambda)\right](x)
$$

Nous utilisons ultérieurement cette définition pour l'étude de l'existence des moments de $p_{\alpha}$. Sous certaines hypothèses, la notion introduite en (2.10) coïncide avec la définition beaucoup plus ancienne donnée par H. Weyl [28] et A. Zygmund [29], [30]. En effet, dans [29], [30], A. Zygmund définit une primitive d'ordre $\gamma>0$ de $u$ par:

$$
P^{\gamma} u(x)=\frac{1}{\Gamma(\gamma)} \int_{-\infty}^{x}(x-t)^{\gamma-1} u(t) d t=\frac{1}{\Gamma(\gamma)}\left(1_{\mathbf{R}_{+}}(t) \times t^{\gamma-1} * u\right)(x) .
$$

Puis, pour $\beta>0$, il pose (Ent désigne la partie entière):

$$
D_{x}^{\beta} u(x)=\frac{d^{\operatorname{Ent}(\beta+1)}}{d x^{\operatorname{Ent}(\beta+1)}}\left[P^{\operatorname{Ent}(\beta+1)-\beta} u\right](x),
$$

où la dérivation d'ordre $p=\operatorname{Ent}(\beta+1): D_{x}^{p}=\frac{d^{p}}{d x^{p}}$, retrouve son sens classique lorsque $p \in \mathbf{N}$. En fait, la définition de la dérivée d'indice non entier donnée par la formule (2.10) est équivalente, dans notre contexte, à celle proposée par H. Weyl et A. Zygmund.

Une première étude des moments d'ordre entiers de $p_{\alpha}$ montre que, pour tout entier $\beta \in \mathbf{N}$, si $h$ admet une dérivée partielle en $\lambda$ d'ordre $\beta$ au sens des distributions tempérées, alors $x^{\beta} p_{\alpha}(x, t)$ admet une transformée de Fourier au sens des distributions tempérées, qui vérifie, lorsque ces distributions sont des fonctions:

$$
\int x^{\beta} p_{\alpha}(x, t) e^{-i a x} d x=(2 \pi)^{\frac{1}{2}} i^{\beta} \frac{\partial^{\beta} h}{\partial \lambda^{\beta}}(a, t) \quad \text { (presque partout) }
$$

Prenant $a=0$, on déduit alors:

THÉorÈmE. Sous les hypothèses précédentes, si $\frac{\partial^{\beta} h}{\partial \lambda^{\beta}}(\bullet, t)$ est une fonction continue et intégrable, alors:

$$
\int x^{\beta} p_{\alpha}(x, t) d x=(2 \pi)^{\frac{1}{2}} i^{\beta} \frac{\partial^{\beta} h}{\partial \lambda^{\beta}}(0, t) .
$$

On peut, par ailleurs, donner leur générateur: pour tout $\alpha$ appartenant à $\mathbf{R}_{+}^{*}$, le générateur infinitésimal du semi-groupe dont le noyau de convolution est $p_{\alpha}(x, t)$ est donné par:

$$
G_{\alpha}=-\frac{\operatorname{sgn}\left[\Re\left(i^{\alpha}\right)\right]}{\Gamma(a+1)} \frac{\partial^{\alpha}}{\partial x^{\alpha}} .
$$

En effet, si nous reprenons la notation $s_{\alpha}=-\operatorname{sgn} \Re\left(i^{\alpha}\right)$, nous avons:

$$
\begin{aligned}
\frac{\partial}{\partial t} \mathcal{F} p & (\bullet, t)(\lambda)=\frac{\partial}{\partial t}(2 \pi)^{-\frac{1}{2}} \exp \left[-s_{\alpha}(i \lambda)^{\alpha} \frac{t}{\Gamma(\alpha+1)}\right]= \\
& -\frac{s_{\alpha}(i \lambda)^{\alpha}}{\Gamma(\alpha+1)}(2 \pi)^{-\frac{1}{2}} \exp \left[-s_{\alpha}(i \lambda)^{\alpha} \frac{t}{\Gamma(\alpha+1)}\right]
\end{aligned}
$$

De (2.10), nous déduisons alors:

$$
\frac{\partial p(x, t)}{\partial t}=-\frac{s_{\alpha}}{\Gamma(a+1)} \frac{\partial^{\alpha}}{\partial x^{\alpha}} p(x, t) .
$$




\section{Etudes préliminaires sur quelques exemples}

Nous examinons quelques cas particuliers qui sous-tendent l'étude ultérieure:

$-\alpha=2$ : cas gaussien. On a alors:

$$
h_{2}(\lambda, t)=(2 \pi)^{-\frac{1}{2}} \exp \left[-\frac{\lambda^{2} t}{2}\right]
$$

et :

$$
p_{2}(x, t)=(2 \pi t)^{-\frac{1}{2}} \exp \left[-\frac{x^{2}}{2 t}\right]
$$

- $\alpha=\frac{1}{2}$ : hyperdiffusion. Cette valeur de $\alpha$ donne

$$
h_{\frac{1}{2}}(\lambda, t)=(2 \pi)^{-\frac{1}{2}} \exp \left[(i \lambda)^{\left(\frac{1}{2}\right)} \frac{2 t}{\sqrt{\pi}}\right]
$$

ce qui conduit, par transformée de Fourier inverse à:

$$
p_{\frac{1}{2}}(x, t)=1_{\mathbf{R}_{+}}(x) x^{-\frac{3}{2}} t 2 \pi^{\frac{1}{2}} \exp \left[-4 \pi^{2} t^{2} / x\right] .
$$

- $\alpha=1$ : une mesure non absolument continue par rapport à celle de Lebesgue, un processus déterministe et un processus de Cauchy; dans ce cas:

$$
p_{1}(d x, t)=\delta_{\{x=-t\}} \text { et } p_{1}(x, t)=\frac{k_{0} t}{\left(x+k_{1} t\right)^{2}+\left(k_{0} t\right)^{2}} ;
$$

$p_{1}$ est la probabilité de transition d'un processus déterministe;

$-\alpha \in 2 \mathbf{N}, \alpha \geq 4$ : hypodiffusions. Les distributions $p_{2 n}(x, t)$ admettent des moments de tous ordres; elles vérifient:

$$
\frac{\partial p_{2 n}}{\partial t}(x, t)=\frac{(-1)^{n+1}}{(2 n) !} \frac{\partial^{2 n} p_{2 n}}{\partial x^{2 n}}(x, t) ;
$$

elles sont paires, du type "hypergaussiennes".

$-\alpha=3$ : hypodiffusion d'Airy. Dans ce cas:

$$
h_{3}(\lambda, t)=(2 \pi)^{-\frac{1}{2}} \exp \left[-i \lambda^{3} t / 3 !\right]
$$

cette fonction vérifie :

$$
(i \lambda)^{2} \operatorname{th}(\lambda)+2 i \frac{\partial h}{\partial \lambda}(\lambda)=0,
$$

et sa transformée de Fourier réciproque est solution de:

$$
\frac{\partial p_{3}}{\partial x^{2}}(x, t)+\frac{2 x}{t} p_{3}(x, t)=0
$$

la fonction $p_{3}$ est une fonction d'Airy:

$p_{3}(x, t)=(2 \pi)^{-1 / 2} \int \exp \left[i\left(\lambda x-\frac{\lambda^{3} t}{3 !}\right)\right] d \lambda=2^{5 / 6} \pi^{1 / 2} t^{-1 / 3} \operatorname{Airy}\left(x^{3} \sqrt{\frac{2}{t}}\right)$,

où:

$$
\operatorname{Airy}(y):=\frac{1}{2 \pi} \int \exp \left[i\left(-y u+\frac{u^{3}}{3}\right)\right] d u .
$$

$-\alpha=5$ : cas d'une autre hypodiffusion. Dans [2], A. Barrantes, A. Calvo et M. Rosen ont mis en évidence une hypodiffusion correspondant à la diffusion d'un traceur (fluoresceïne) dans de l'eau (les coefficients qui apparaissent dans les différents phénomènes sont alors $\alpha=3 / 2$ et $\alpha=5$ ). Ici, $p_{5}$ n'apparait plus comme une fonction d'Airy; nous l'étudions dans la partie 3 . 


\section{Etude asymptotique des solutions dans le cas où l'ordre de DÉRIVATION EST UN ENTIER IMPAIR}

L'objectif est de déterminer des estimées asymptotiques pour $x \rightarrow+\infty$ des solutions de l'équation:

$$
\frac{\partial u}{\partial t}=c \frac{\partial^{2 n+1} u}{\partial x^{2 n+1}},
$$

avec $n \geq 2, t>0, x$ réel et condition initiale $u(x, 0)=\delta(x)$. Par transformation de Fourier,

$$
u(x, t)=\frac{1}{2 \pi} \int \exp \left(i \alpha x+c(i \alpha)^{2 n+1} t\right) d \alpha .
$$

On pose alors $\alpha=\left(\frac{x}{t}\right)^{\frac{1}{2 n}} z$; alors:

$$
u(x, t)=\frac{1}{2 \pi}\left(\frac{x}{t}\right)^{\frac{1}{2 n}} \int \exp \left(i \lambda\left(z+c(-1)^{n} z^{2 n+1}\right)\right) d z \text { avec } \lambda=x^{1+\frac{1}{2 n}} t^{-\frac{1}{2 n}} .
$$

On va étudier la convergence de cette intégrale. Pour cela on considère:

$$
I_{s, t}=\int_{s}^{t} \sin \left(\lambda\left(x+c(-1)^{n} x^{2 n+1}\right)\right) d x
$$

par changement de variable $y=x+c(-1)^{n} x^{2 n+1}$, on obtient $x=g(y)$ avec $g$ une fonction croissante qui tend vers l'infini. Il vient:

$$
g(y)=\frac{1}{1+(2 n+1) c(-1)^{n} g^{2 n}(y)},
$$

donc:

$$
I_{s, t}=\int_{s+c(-1)^{n} s^{2 n+1}}^{t+c(-1)^{n} t^{2 n+1}} \frac{\sin (\lambda y) d y}{1+(2 n+1) c(-1)^{n} g^{2 n}(y)} ;
$$

par changement de variables $u=\lambda y, I_{s, t}$ tend vers:

$$
I_{s, t} x \rightarrow+\infty \rightarrow I=\frac{1}{\lambda} \int_{0}^{+\infty} \frac{\sin u}{1+(2 n+1) c(-1)^{n} g^{2 n}\left(\frac{u}{\lambda}\right)} d u
$$

or il existe $\sigma>0$ tel que pour tous $c$ et $d,\left|\int_{c}^{d} \sin u d u\right|<\sigma$ et $\frac{1}{1+(2 n+1) c(-1)^{n} g^{2 n}\left(\frac{u}{\lambda}\right)}$ est décroissante (ou croissante suivant le signe de $(-1)^{n}$ ) vers 0 , donc l'intégrale de départ converge.

On considère :

$$
I(\lambda)=\int \exp (\lambda f(z)) d z \text { avec } f(z)=i z+i c(-1)^{n} z^{2 n+1} ;
$$

donc:

$$
f^{\prime}(z)=i+(2 n+1) i c(-1)^{n} z^{2 n} .
$$

Les points selle sont tels que $f^{\prime}(z)=0$, ce qui équivaut à $z^{2 n}=-\frac{1}{(2 n+1) c(-1)^{n}}$.

$$
\text { 4.1. CAS : } c(-1)^{n}<0
$$

On a alors les points selle suivants:

$$
z_{p}=\left(\frac{-1}{(2 n+1) c(-1)^{n}}\right)^{\frac{1}{2 n}} \exp \left(\frac{(p+1) i \pi}{\pi}\right), \text { avec } p=0,1, \ldots, 2 n-1 .
$$

Lemme. On a l'estimation asymptotique:

$$
\begin{aligned}
& I(\lambda) \approx \sqrt{\frac{\pi}{2 \lambda\left|f^{\prime \prime}\left(z_{n-1}\right)\right|}}\left(\exp \left(\lambda f\left(z_{n-1}\right)\right)\right)\left(\exp \left(i \theta_{0}\right)-\exp \left(i \theta_{1}\right)\right) \\
& +\sqrt{\frac{\pi}{2 \lambda\left|f^{\prime \prime}\left(z_{2 n-1}\right)\right|}\left(\exp \left(\lambda f\left(z_{2 n-1}\right)\right)\right)\left(\exp \left(i \theta_{0}^{\prime}\right)-\exp \left(i \theta_{1}^{\prime}\right)\right)}
\end{aligned}
$$


Preuve. On donne les étapes principales. Davantage de détails peuvent être trouvés en [23]. On calcule $f\left(z_{p}\right)$ et on considére ceux qui ont une partie réelle négative ou nulle; parmi ceux-ci, on ne gardera que ceux qui ont la plus grande partie réelle.

On a:

Donc:

$$
\begin{gathered}
f\left(z_{p}\right)=i\left(\frac{-1}{(2 n+1) c(-1)^{n}}\right)^{\frac{1}{2 n}} \exp \left(\frac{(p+1) i \pi}{n}\right) \\
+i c(-1)^{n}\left(\frac{-1}{(2 n+1) c(-1)^{n}}\right)^{1+\frac{1}{2 n}} \exp \left(\frac{(p+1) i \pi}{n}\right) .
\end{gathered}
$$

$$
\Re\left(f\left(z_{p}\right)\right)=\left(\frac{-2 n}{2 n+1}\right)\left(\frac{-1}{(2 n+1) c(-1)^{n}}\right)^{\frac{1}{2 n}} \sin \left(\frac{(p+1) \pi}{n}\right) .
$$

On ne considère que les $z_{p}$ pour lesquels $\sin \left(\frac{(p+1) \pi}{n}\right)=0$, c'est à dire $p=n-1$ et $p=2 n-1$.

- Cas du premier point-selle: $z_{n-1}$. On a après calcul:

$$
f^{\prime \prime}\left(z_{n-1}\right)=2 n\left(\frac{-1}{(2 n+1) c(-1)^{n}}\right)^{-\frac{1}{2 n}} \exp \left(\frac{i \pi}{2}\right)=a \exp (i \varphi)
$$

les chemins de plus grande pente ont donc les directions suivantes, caractérisées par l'angle, avec l'axe réel, de la tangente au chemin de plus grande pente au point selle considéré, noté $\theta_{k}$ (voir Bleistein-Handelsman (1986)):

$$
\theta_{k}=-\frac{\pi}{4}+(2 k+1) \frac{\pi}{2}, k=0,1 .
$$

- cas du second point-selle: $z_{2 n-1}$. On a:

$$
f^{\prime \prime}\left(z_{2 n-1}\right)=2 n\left(\frac{-1}{(2 n+1) c(-1)^{n}}\right)^{\frac{-1}{2 n}} \exp \left(\frac{3 i \pi}{2}\right) ;
$$

les chemins de plus grande pente ont les directions suivantes (voir BleisteinHandelsman (1986)):

$$
\theta_{k}^{\prime}=-\frac{3 \pi}{4}+(2 k+1) \frac{\pi}{2}, k=0,1 .
$$

On pose:

$$
\xi=\left(\frac{2 n}{2 n+1}\right)\left(\frac{-1}{(2 n+1) c(-1)^{n}}\right)^{\frac{1}{2 n}}
$$

on a alors : $f\left(z_{n-1}\right)=-i \xi$ et $f\left(z_{2 n-1}\right)=i \xi$. Après calculs,

ThÉorÈme. On a l'estimation asymptotique:

$$
\begin{gathered}
u(x, t) \approx \frac{\sqrt{2}}{\pi}\left(\frac{x}{t}\right)^{\frac{1}{2 n}} \sqrt{\frac{\pi}{2 x^{1+\frac{1}{2 n}} t^{-\frac{1}{2 n}}\left|f^{\prime \prime}\left(z_{n-1}\right)\right|}} \\
\times\left(\cos \left(\xi x^{1+\frac{1}{2 n}} t^{-\frac{1}{2 n}}\right)+\sin \left(\xi x^{1+\frac{1}{2 n}} t^{-\frac{1}{2 n}}\right)\right) . \\
\text { 4.2. CAS } c(-1)^{n}>0 .
\end{gathered}
$$

Comme précédemment, nous utilisons le lemme 4.1. il vient:

$$
z_{p}=\left(\frac{1}{(2 n+1) c(-1)^{n}}\right)^{\frac{1}{2 n}} \exp \left(\frac{(2 p+1) i \pi}{2 n}\right) \text { avec } p=0,1, \ldots, 2 n-1 ;
$$

on en déduit:

$$
\Re\left(f\left(z_{p}\right)\right)=\left(\frac{-2 n}{2 n+1}\right)\left(\frac{1}{(2 n+1) c(-1)^{n}}\right)^{\frac{1}{2 n}} \sin \left(\frac{(2 p+1) \pi}{2 n}\right) .
$$


On ne considère que les points pour lesquels la partie réelle est négative tout en étant la plus grande possible donc on ne prend en compte que $z_{0}$ et $z_{n-1}$.

- Cas du premier point-selle $z_{0}$. On a:

$f^{\prime \prime}\left(z_{0}\right)=2 n(2 n+1) i c(-1)^{n} z_{0}^{2 n-1}=2 n\left(\frac{1}{(2 n+1) c(-1)^{n}}\right)^{-\frac{1}{2 n}} \exp \left(\frac{(3 n-1) i \pi}{2 n}\right) ;$

les chemins de plus grande pente ont les directions:

$$
\theta_{k}=-\left(\frac{3 n-1}{2 n}\right) \frac{\pi}{2}+(2 k+1) \frac{\pi}{2}, k=0,1 .
$$

- Cas du second point-selle $z_{n-1}$. On a:

$$
f^{\prime \prime}\left(z_{n-1}\right)=2 n\left(\frac{1}{(2 n+1) c(-1)^{n}}\right)^{-\frac{1}{2 n}} \exp \left(\left(\frac{4 n^{2}+n+1}{2 n}\right) i \pi\right) ;
$$

les chemins de plus grande pente ont les directions:

$$
\theta_{k}^{\prime}=-\left(\frac{4 n^{2}+n+1}{2 n}\right) \frac{\pi}{2}+(2 k+1) \frac{\pi}{2}, k=0,1 \text {. }
$$

$\diamond$

En posant :

$$
\xi=\left(\frac{2 n}{2 n+1}\right)\left(\frac{1}{(2 n+1) c(-1)^{n}}\right)^{\frac{1}{2 n}},
$$

on montre finalement d'après les lemmes précédents:

THÉorème. On a l'estimation asymptotique:

$$
\begin{aligned}
& u(x, t) x \rightarrow+\infty \approx \frac{2}{\pi}\left(\frac{x}{t}\right)^{\frac{1}{2 n}} \sqrt{\frac{\pi}{2 x^{1+\frac{1}{2 n}} t^{-\frac{1}{2 n}}\left|f^{\prime \prime}\left(z_{0}\right)\right|}} \\
& \times\left(\exp \left(\xi x^{1+\frac{1}{2 n}} t^{-\frac{1}{2 n}} \cos \left(\frac{(n+1) \pi}{2 n}\right)\right)\right) \\
& \times \cos \left(\xi x^{1+\frac{1}{2 n}} t^{-\frac{1}{2 n}} \sin \left(\frac{(n+1) \pi}{2 n}\right)+\frac{\pi}{4 n}-\frac{\pi}{4}\right) .
\end{aligned}
$$

Remarque. Ces estimations asymptotiques sont valables pour $x \rightarrow+\infty$; pour $x \rightarrow-\infty$, il suffit de changer le signe de $c$. Donc, pour déterminer les estimations pour $c<0$ et $x \rightarrow-\infty$, il suffit de prendre les solutions pour $c>0$ et $x \rightarrow+\infty$, et de remplacer $x$ par $|x|$. De même pour $c>0$ et $x \rightarrow-\infty$, il suffit de considérer les estimations pour $c<0$ et $x \rightarrow+\infty$, et de remplacer $x$ par $|x|$.

\section{ETUdE Asymptotique DES SOLUTIONS DANS le CAS OÙ L'ORDRE DE DÉRIVATION EST FRACTIONNAIRE.}

L'objectif est de déterminer des estimées asymptotiques des solutions de l'équation:

avec $\beta=\frac{3}{2}, t>0$, et $x$ réel.

$$
\frac{\partial u}{\partial t}=c \frac{\partial^{\beta} u}{\partial x^{\beta}}
$$

LEMME. On a l'estimation:

$$
I(\lambda) \approx \frac{4}{3} \sqrt{\frac{\pi}{\lambda}} \exp \left(\frac{-4 \lambda}{9}+i\left(\frac{\pi}{4}-\frac{8 \lambda}{27}\right)\right) .
$$

THÉorème. On a l'estimation asymptotique:

$$
u(x, t) x \rightarrow+\infty \approx \frac{2}{3 \pi}\left(\frac{x}{t}\right)^{2} \sqrt{\frac{\pi}{x^{3} t^{-2}}} \exp \left(-\frac{4 x^{3} t^{-2}}{9}+i\left(\frac{\pi}{4}-\frac{8 x^{3} t^{-2}}{27}\right)\right)
$$


Preuve. On obtient par transformation de Fourier:

$$
u(x, t)=\frac{1}{2 \pi}\left(\frac{x}{t}\right)^{\frac{1}{\beta-1}} \int \exp \left(i \lambda\left(z+c i^{\beta-1} z^{\beta}\right)\right) d z \text { avec } \lambda=x^{\frac{\beta}{\beta-1}} t^{-\frac{1}{\beta-1}} .
$$

On considère :

$$
I(\lambda)=\int \exp (\lambda f(z)) d z \text { avec } f(z)=i z+c i^{\beta} z^{\beta}
$$

on n'a qu'un seul point selle:

$$
z_{0}=\left(\frac{1}{\beta c}\right)^{\frac{1}{\beta-1}} e^{i \frac{\pi}{2}}
$$

on prend $c=1$ et donc: $\Re\left(f\left(z_{0}\right)\right)=-\frac{4}{9}$. Les chemins de plus grande pente sont tels que:

$$
\theta_{k}=-\frac{\pi}{4}+(2 k+1) \frac{\pi}{2} \text { avec } k=0,1
$$

Ce résultat peut être étendu à d'autres valeurs de $\beta$ moyennant quelques adaptations techniques (voir [23]).

\section{Etude d’une distribution $\mathbf{P}_{\alpha}$ sur un espace fonctionnel \\ LORSQUE $\alpha>1$, AVEC $\alpha \notin 2 \mathrm{~N}+1$}

Dans ce paragraphe, partant des distributions-mesures $p(., t)=p_{\alpha}(., t)$, nous allons, par "limite projective", construire une distribution sur l'espace $\Omega$ des fonctions continues à droite avec existence de limite à gauche (c.a.d.l.a.g):

$$
\Omega=\left\{\omega: \mathbf{R}_{+} \rightarrow \mathbf{R}, t \rightarrow \omega(t), \omega \text { c.a.d.l.a.g., } \omega(0)=0\right\} .
$$

Cette distribution généralisera la mesure de Wiener; c'est une mesure positive lorsque $\alpha \leq 2$, et une mesure signée si $\alpha>2$.

Les éléments de $\Omega$ sont, suivant l'usage, appelés "trajectoires".

On considère l'ensemble des parties finies de $\mathbf{R}_{+}$, ordonné par l'inclusion, et, pour tout $\tau=\left\{t_{1}<t_{2}<\ldots<t_{n}\right\}$ :

$$
\begin{aligned}
& \xi_{\tau}: \Omega \rightarrow \mathbf{R}^{n} \\
& \omega \rightarrow\left(\omega\left(t_{1}\right), \ldots, \omega\left(t_{n}\right)\right) .
\end{aligned}
$$

On note $\mathcal{F}_{\tau}=\xi_{\tau}^{-1}\left(\mathcal{B}\left(\mathbf{R}^{n}\right)\right)$ l'image réciproque de la tribu borélienne de $\mathbf{R}^{n}$ par cette application $\xi_{\tau}$ et on designe par $\mathcal{F}$ la tribu engendrée par $\mathcal{F}_{\tau}$ lorsque $\tau$ décrit les parties finies de $\mathbf{R}_{+}$.

Notant $S\left(\mathbf{R}^{n}\right)$ l'espace des fonctions définies sur $\mathbf{R}^{n}$ et à valeurs complexes, bornées et uniformément continues, transformées de Fourier de mesures bornées, on désigne par $C:=C(\Omega)$ la classe de fonctions sur $\Omega$ :

$$
C(\Omega)=\left\{f: \Omega \rightarrow \underset{ }{\mathbf{R} ; \exists n \in \mathbf{N}^{*}, \exists t_{1}<t_{2}<\ldots<t_{n}, t_{i} \in \mathbf{R}_{+}, \exists F \in S\left(\mathbf{R}^{n}\right),},\right.
$$

et sur $C$, on définit l'application:

$$
\begin{gathered}
\mathbf{E}_{\alpha}(f)=\mathbf{P}_{\alpha}(f)=\mathbf{P}(f)=\int_{x_{1} \in \mathbf{R}} \int_{x_{2} \in \mathbf{R}} \ldots \int_{x_{n} \in \mathbf{R}} F\left(x_{1}, \ldots, x_{n}\right) \\
p_{\alpha}\left(d\left(x_{n}-x_{n-1}\right), t_{n}-t_{n-1}\right) \ldots p_{\alpha}\left(d\left(x_{2}-x_{1}\right), t_{2}-t_{1}\right) p_{\alpha}\left(d x_{1}, t_{1}\right) \\
=\int_{\lambda \in \mathbf{R}^{n}}^{\mathcal{F}^{-1} F(\lambda) \bar{h}_{\alpha}\left(\lambda_{n}, t_{n}-t_{n-1}\right) \bar{h}_{\alpha}\left(\lambda_{n}+\lambda_{n-1}, t_{n-1}-t_{n-2}\right) \ldots} \\
\ldots \bar{h}_{\alpha}\left(\lambda_{n}+\ldots+\lambda_{1}, t_{1}\right) d \lambda,
\end{gathered}
$$

où $\bar{h}_{\alpha}(\lambda, t)$ désigne le conjugué dans $\mathbf{C}$ de $h_{\alpha}(\lambda, t), \mathbf{P}(f)$ ne dépend que de $f$ et non du choix de $F$ : on voit de plus que $P$ est linéaire sur $C(\Omega)$. 
Pour $\alpha>1, \alpha \notin 2 \mathrm{~N}+1, P$ est une mesure signée additive; elle se définit sur l'algèbre $A$ de $P(\Omega)$, engendrée par les cylindres en posant, pour tout cylindre:

$$
\begin{gathered}
C=\left\{\omega: a_{i} \leq \omega\left(t_{i}\right) \leq b_{i}, i=1,2, \ldots n, 0<t_{1}<t_{2} \ldots<t_{n}\right\} \\
P_{\alpha}(C)=\int_{a_{1}}^{b_{1}} \ldots \int_{a_{n}}^{b_{n}} \prod_{i=1}^{n} p_{\alpha}\left(d x_{i}-d x_{i-1}, t_{i}-t_{i-1}\right), \text { avec } x_{0}=0 \text { et } t_{0}=0 .
\end{gathered}
$$

Nous voyons que la variation totale de $\mathbf{P}$ sur $\Omega$ est:

- bornée ( $\mathbf{P}$ est une probabilité) si $p_{\alpha}(., t)$ est une densité positive $(\alpha \leq 2)$,

- non bornée si $p_{\alpha}(., t)$ n'est pas positive $(\alpha>2)$.

Nous allons définir un prolongement de $\mathbf{P}=\mathbf{P}_{\alpha}$ sur un espace fonctionnel. L'espace considéré sera un sur-ensemble de $C(\Omega)$ (nous procédons par prolongement à partir de $C(\Omega))$.

Si $u \in C(\Omega)$ :

$$
u(\omega)=U\left(\omega\left(t_{1}\right), \omega\left(t_{2}\right), \ldots \omega\left(t_{n}\right)\right), U \in S\left(\mathbf{R}^{\left\{t_{1}, \ldots, t_{n}\right\}}=\mathbf{R}^{n}\right)
$$

nous posons:

$$
\|u\|_{\alpha}=\int_{\lambda \in \mathbf{R}^{n}} d\left|\mathcal{F}^{-1} U(\lambda)\right|\left|h_{\alpha}\left(\lambda_{n}, t_{n}-t_{n-1}\right) \ldots h_{\alpha}\left(\lambda_{n}+\ldots+\lambda_{1}, t_{1}\right)\right| .
$$

Il est clair que $:\left(u \rightarrow\|u\|_{\alpha}\right)$ est une norme sur $C(\Omega)$. Pour toute fonction $u \in C(\Omega)$ : $\left|\mathbf{P}_{\alpha}(u)\right| \leq\|u\|_{\alpha}$. Notant $D_{\alpha}=D_{\alpha}(\Omega)$ le complété de $C(\Omega)$ pour la norme $\|.\|_{\alpha}, \mathbf{P}_{\alpha}$ se prolonge continûment sur $\left(D \alpha(\Omega),\|.\|_{\alpha}\right)$ : si $f \in D_{\alpha}(\Omega)$, alors $f=\lim f_{n}$ où $f_{n}$ est une suite de fonctions appartenant à $C$, et l'on a :

$$
\mathbf{P}_{\alpha}(f)=\lim _{(n \rightarrow \infty)} \mathbf{P}_{\alpha}\left(f_{n}\right)
$$

lorsque $\alpha=2$, on vérifie que:

$$
\begin{gathered}
\|u\|_{2}=\int_{\lambda \in \mathbf{R}^{n}}(2 \pi)^{-n / 2} d\left|\mathcal{F}^{-1} U(\lambda)\right| \exp \left[-\lambda_{n}^{2}\left(t_{n}-t_{n-1}\right) / 2\right] \ldots \\
\times \exp \left[-\left(\lambda_{n}+\ldots+\lambda_{1}\right)^{2} t_{1} / 2\right] .
\end{gathered}
$$

Ceci conduit à définir la norme suivante sur l'espace des mesures bornées sur $\mathbf{R}^{n}$ :

$$
\begin{gathered}
\|\mu\|_{2}=\int_{\lambda \in \mathrm{R}^{n}}(2 \pi)^{-n / 2} \exp \left[-\lambda_{n}^{2}\left(t_{n}-t_{n-1}\right) / 2\right] \ldots \\
\quad \times \exp \left[-\left(\lambda_{n}+\ldots+\lambda_{1}\right)^{2} t_{1} / 2\right] d|\mu|\left(\lambda_{1}, \ldots, \lambda_{n}\right) .
\end{gathered}
$$

On a alors: $\|u\|_{2}=\left\|\mathcal{F}^{-1} U\right\|_{2}$. L'espace $D_{2}(\Omega)$ est donc l'image par $\mathcal{F}^{-1}$ du complété de l'espace des mesures bornées par $\|\bullet\|_{2}$.

\section{Etude particulière de $D_{2}(\Omega)$}

Pour toute fonction $u: \Omega \rightarrow \mathrm{C}$ dans $C(\Omega)$, nous avons posé, si: $u(\omega)=$ $U\left(\omega\left(t_{1}\right), \ldots, \omega\left(t_{n}\right)\right)$ et $\tau=\left\{t_{1}, t_{2}, \ldots, t_{n}\right\}$ :

$$
\|u\|_{2}=\int_{\lambda \in \mathbf{R}^{n}} d\left|\mathcal{F}^{-1} U(\lambda)\right| . h_{2}\left(\lambda_{n}, t_{n}-t_{n-1}\right) \ldots h_{2}\left(\lambda_{n}+\ldots .+\lambda_{1}, t_{1}\right) .
$$

Nous pouvons obtenir une majoration de $\|u\|_{2}$ par la masse totale de $\mathcal{F}^{-1} U$. Si $\mu$ est une mesure bornée sur $\mathbf{R}^{n}$, nous notons ||$|\mu|||$ sa masse totale:

$$
\left\|\left|\mu \|=(2 \pi)^{-n / 2} \int_{\lambda \in \mathbf{R}^{n}} d\right| \mu(\lambda) \mid .\right.
$$

Puisque $h_{2}$ a une valeur comprise entre 0 et $(2 \pi)^{-1 / 2}$, on a:

$$
\|u\|_{2} \leq\left\|\mathcal{F}^{-1} U\right\| \| \text {. }
$$


Nous allons maintenant comparer $\|u\|_{2}$ et les normes de $u$ dans $L^{1}(P)$ et $L^{2}(P)$. Nous pouvons tout d'abord remarquer que:

$$
\|u\|_{L^{1}(P)} \leq\|\| \mathcal{F}^{-1} U\|\|
$$

en effet,

$$
\left\|\left|\mathcal{F}^{-1} U\right|\right\|=\sup .\left\{(2 \pi)^{-n / 2}\left(\|F\|_{\infty}\right)^{-1} \int_{x \in \mathbf{R}^{n}} \mathcal{F} F(x) U(x) d x\right\}
$$

En prenant:

$$
F(\lambda)=h_{2}\left(\lambda_{n}, t_{n}-t_{n-1}\right) \ldots h_{2}\left(\lambda_{n}+\ldots+\lambda_{1}, t_{1}\right),
$$

nous obtenons, dans la formule précédente, $\|F\|_{\infty}=(2 \pi)^{-n / 2}$; par suite:

$$
\begin{gathered}
\quad\left\|\mathcal{F}^{-1} U \mid\right\| \geq \int_{x \in \mathbf{R}^{n}} U(x) \cdot p_{2}\left(d x_{n}-x_{n-1}, t_{n}-t_{n-1}\right) \ldots p_{2}\left(d x_{1}, t_{1}\right) \\
\geq \int_{x \in \mathbf{R}^{n}}|U(x)| \cdot p_{2}\left(d x_{n}-d x_{n-1}, t_{n}-t_{n-1}\right) \ldots p_{2}\left(d x_{1}, t_{1}\right)=\|u\|_{L^{1}(P)} .
\end{gathered}
$$

Proposition. $D_{2} \not \subset L^{2}(P)$.

Preuve. Nous donnons un exemple de suite $\left(u_{n}\right) \in C(\Omega)$ telle que $\left\|u_{n}\right\|_{2} \rightarrow 0$ et $\left\|u_{n}\right\|_{L^{2}(P)} \not \rightarrow 0$. Considérons:

$$
\psi(x)=c \times 1_{[a, b]}(x) ;
$$

on a alors:

$$
\begin{gathered}
\mathcal{F}^{-1} \psi(\lambda)=\left\{\begin{array}{l}
(2 \pi)^{-1 / 2} \frac{\sin [\lambda \cdot(b-a) / 2]}{\lambda} e^{i \lambda(a+b) / 2}, \lambda \neq 0 \\
\frac{c(b-a)}{(2 \pi)^{1 / 2}}, \lambda=0,
\end{array}\right. \\
\left|\mathcal{F}^{-1} \psi(\lambda)\right| \leq \frac{|c| .(b-a)}{(2 \pi)^{1 / 2}} \text { avec égalité si } \lambda=0 .
\end{gathered}
$$

Nous imposons la condition $\frac{|c| \cdot(b-a)}{(2 \pi)^{1 / 2}}<1$ et considérons maintenant la suite $\left(u_{n}\right)_{n \in \mathbf{N}}$, définie par:

$$
u_{n}(\omega)=\psi(\omega(1)) \cdot \psi(\omega(2)-\omega(1)) \ldots \psi(\omega(n)-\omega(n-1)),
$$

ce qui correspond à: $U_{n}(x)=\psi\left(x_{1}\right) \cdot \psi\left(x_{2}-x_{1}\right) \ldots \psi\left(x_{n}-x_{n-1}\right)$, et aux temps $t_{1}=1, t_{2}=2, \ldots, t_{n}=n$. La fonction $u_{n}$ n'appartient pas à $C(\Omega)$, mais elle est évidemment dans $D_{2}$ et on peut montrer que:

$$
\left\|u_{n}\right\|_{2} \rightarrow 0 \text { lorsque } n \rightarrow \infty \text {. }
$$

Par conséquent:

$$
u_{n} \in D_{2} \text { et }\left(u_{n}\right)_{n \in \mathbf{N}} \rightarrow 0, \text { dans } D_{2}
$$

Par contre:

$$
\left\|u_{n}\right\|_{L^{2}(P)} \geq\left((2 \pi)^{-1 / 2} c^{2} e^{-b^{2} / 2}(b-a)\right)^{n / 2},
$$

si $|a| \leq|b|$. On peut choisir $a, b$ et $c$ de manière que soient vérifiées simultanément les trois conditions:

$$
|a| \leq|b|, \frac{|c| .(b-a)}{(2 \pi)^{1 / 2}}<1 \text {, et }(2 \pi)^{-1 / 2} c^{2} e^{-b^{2} / 2}(b-a)>1 .
$$

Pour un tel choix de $(a, b, c)$, on a $\left\|u_{n}\right\|_{L^{2}(P)} \rightarrow \infty$. 


\section{Etude générale des moments}

Nous avons vu que, au sens de la transformation de Fourier des distributions tempérées, si $\beta \in \mathbf{N}$, et si $h$ admet une dérivée partielle en $\lambda$ d'ordre $\beta$, alors

$$
\int_{\mathbf{R}} x^{\beta} p_{\alpha}(x, t) d x=(2 \pi)^{1 / 2} i^{\beta} \frac{\partial^{\beta} h_{\alpha}}{\partial \lambda^{\beta}}(0, t)
$$

Lorsque $h_{\alpha}$ admet une dérivée en 0 à l'ordre $n \in \mathrm{N}$, alors $h_{\alpha}^{(n)}(., t)(t>0)$ appartient à tous les espaces $L^{p}, 1 \leq p \leq \infty$ et l'on a: $\left\|x^{n} p_{\alpha}(x, t)\right\|_{\infty} \leq\left\|h_{\alpha}^{(n)}(t)\right\|_{1}$.

Proposition. Pour tout réel $\alpha \in \mathbf{R}_{+}^{*} \backslash(2 \mathbf{N}+1)$, et tout réel $\beta \in[0$, Ent $\alpha[$, $x^{\beta} p_{\alpha}(x, t)$ est intégrable.

Preuve. Si nous notons $n=$ Ent $\alpha, h_{\alpha}(., t)$ est $n$ fois continûment dérivable et $h_{\alpha}^{(n)}(., t) \in L^{p}(\mathbf{R})$ pour tout $p \in[1, \infty[$. Utilisant le théorème de Hausdorff-Young, nous savons que sa transformée de Fourier, $i^{n} x^{n} p_{\alpha}(x, t)$ appartient à $L^{q}(\mathbf{R})$, pour $\frac{1}{p}+\frac{1}{q}=1$. Nous choisissons $p=\frac{n-\beta+1}{n-\beta}$ et $q=n-\beta+1$. Nous avons alors:

$$
\begin{gathered}
\int_{\mathbf{R} \backslash[0,1]}|x|^{\beta}\left|p_{\alpha}(x, t)\right| d x \leq \\
{\left[\int_{\mathbf{R} \backslash[0,1]}\left|x^{n} p_{\alpha}(x, t)\right|^{q} d x\right]^{1 / q}\left[\int_{\mathbf{R} \backslash[0,1]}|x|^{(\beta-n) p} d x\right]^{1 / p} .}
\end{gathered}
$$

La seconde intégrale du produit est convergente dès que $(\beta-n)<-1$, ainsi que la première $\left(\operatorname{car} x^{n} p_{\alpha}(x, t) \in L^{q}\right)$. Or $p_{\alpha}$ est de classe $C^{\infty}$, par conséquent, $x^{\beta} p_{\alpha}(x, t)$ est localement intégrable, ce qui permet de conclure.

Ce résultat peut être étendu à $\alpha \in \mathbf{R}_{+}^{*} \backslash(2 \mathbf{N}+1), \beta \in[0, \alpha[$.

Remarque. Pour les fonctions positives, on a en revanche: $\|u\|_{L^{1}(P)} \leq\|u\|_{2}$.

\section{RÉFÉRENCES}

[1] G. ACCETA, E. ORSINGHER. Asymptotic expansion of fundamental solutions of higher order heat equation. Random oper. and Stoch. Equations, vol. 3, pp 217-226 (1997).

[2] A. BARRANTES, A. CALVO, M. ROSEN et J.E. WESFREID. Velocity field structure and semiquantative analysis of tracer dispersion in a Taylor-Vortex flow of wide gap. Kluwer Acad. Publish. Netherlands (1991).

[3] J. BERNSTEIN. Uber das Fourier integral $\int_{0}^{\infty} e^{-x^{4}} \cos (t x) d x$, Math. Ann. 79, pp 265-268 (1919).

[4] N. BLEISTEIN and A. HANDELSMAN. A symptotic expansion of integrals. Dover, New York (1986).

[5] W.R. BURWELL. Asymptotic expansion of generalized hypergeometric functions, proc Lond. Math. Soc. 22, 57-72 (1923).

[6] Yu L. DALETSKY. Functional integrals connected with operator evolution equations, Russian Mathematical surveys, 1-107 (1962).

[7] Yu L. DALETSKY and S.V. Fomin. Generalized measures in function spaces, Theory of Probability and its applications, 10, 304-316 (1965).

[8] Yu L. DALETSKY. Integration in function spaces, Progress in mathematics, (Ed. R.V. Gamkrelidze) vol. 4, 86-132 (1969.

[9] M. GIONA, H.E. ROMAN. Fractional diffusion equation on fractals: one dimensional case, an asympotic behaviour. J. Phys. A: Math.Gene. 25 , p. 2095-2105 (1992).

[10] B.V GNEDENKO, A.N. KOLMOGOROV. Limit distributions for sums of independent random variables, Addison-Wesley Publishing Compagny (1954).

[11] R. GORENFLO. Fractional calculus: some numerical methods, Fractal and fractional calculus in Continuum Mechanics, pp 277-290.

[12] R. GORENFLO and F. MAINARDI. Fractional calculus and stable probability distributions, Arch. Mechanics, (1998) (in press).

[13] K.J. HOCHBERG. A signed measure on path space related to Wiener measure. Annals of Probability, vol. 6, p. 433-458 (1978). 
[14] K.J. HOCHBERG and E. ORSINGHER. The arc-sine law and its analogs for processes governed by signed and complex measures, Stochastic Processes and their applications, 52, 273-292 (1994).

[15] V. Y. KRYLOV. Some properties of the distribution corresponding to the equation. Soviet Math. Dokl 1, p. 760-763 (1960).

[16] V.Y. KRILOV. Integration of analytic functionals on sign-alternating distributions, Dokl. Akad. Nauk SSSR, 163, 2, 289-292 (1965).

[17] V.I. LADOKIN. Generalization of some theorems of probability theory in the case of complex distributions, Uch. Zap. Kazan. Univ. 125, 6, 94-100 (1965).

[18] V.I. LADOKHIN. On nonpositive distributions, Uch. Zap. Kazan. Univ. 122, 4, 53-64 (1962).

[19] P. LEVY. Sur une application de la dérivée d'ordre non entier au calcul des probabilités, C.R. Acad. Sci., 176, 1118-1120 (1923).

[20] P. LEVY. Théorie de l'addition des variables aléatoires, Gauthier-Villars, Paris (1954).

[21] LIGHTHILL. Fourier analysis and generalized functions. Stundent's edition, Cambridge University Press (1958-1978).

[22] F. MAINARDI. The fundamental solutions for the fractional diffusion-wave equation, Applied Matthematics Letters, 9, no 6, 23-28 (1996).

[23] M. MASTRANGELO. Internal note (1998).

[24] E. ORSINGHER. Processes governed by signed measures connected with third order "heattype" equations, Lithuanian Mathematical Journal 31, 220-231 (1991).

[25] G. POLYA. On zeroes of an integral function represented by Fourier's integral, Messenger Math., 52, 185-188 (1923).

[26] L.F. RICHARDSON. Atmospheric diffusion shown on a distance neighbour graph. Proc. Roy. Soc. London., All 0, 709 (1926).

[27] P. TABELING, O. CARDOSO. Diffusion anormale: un phénomène presque banal en hydrodynamique, Bull de la Société francaise de Physique, no 73 (Juillet 1989).

[28] H. WEYL. Bemerkungen zum Begriff des Différentialquotienten gebrochener Ordnung, Vierteljschr. Naturforsch. Gesellsch. Zûrich 62, 296 (1917).

[29] A. ZYGMUND. Trigonometrical Series, Vol 2, Cambridge University Press, Cambridge (1935-1959).

[30] A ZYGMUND. Theorem on fractionnal derivatives. Duke Math J. 12, p. 455-464 (1945). 
